\title{
Lessons Learnt From Managing Delayed Case of Primary Hyperparathyroidism in a Tertiary Care Centre in India
}

\author{
Dr. Sumanta Kumar Mishra ${ }^{1}$, Dr kailash Chandra mohapatra ${ }^{2,}$ \\ Dr Sumit S Mohanty ${ }^{3,}$ Dr Manas R Samantray ${ }^{4}$ \\ 1,2,3,4 (Dept. of General Surgery, S.C.B Medical College, Cuttack, India)
}

\begin{abstract}
The disease spectrum of primary hyperparathyroidism varies from asymptomatic hypercalcemia to severe metabolic bone disease, pathological fractures and renal stones. Lack of universal screening for hypercalcemia and lack of awareness about prevalence and presentation of PHPT among general practitioners led to delayed diagnosis. With this study we emphasise on how improving early detection of PHPT the dreadly complications of this curable disease can be avoided.

Key Words: Asymptomatic hypercalcemia,early detection, screening
\end{abstract}

\section{Introduction}

Primary hyperparathyroidism is an endocrine disorder due to excessive uncontrolled secretion of parathyroid hormone from one or more parathyroid glands. Incidence of PHPT was estimated to be 20 per $100,000{ }^{[1,2]}$ and more than $80 \%$ of PHPT cases is asymptomatic at the time of diagnosis ${ }^{[3,4]}$. It presents with variable clinical expression, frequently as asymptomatic hypercalcemia in western countries but still predominantly as a symptomatic disease in developing countries. The disease spectrum of primary hyperparathyroidism varies from asymptomatic hypercalcemia to severe metabolic bone disease, pathological fractures and renal stones. Previous Indian studies show greater severity of primary hyperparathyroidism due to delayed diagnosis.

\section{Aims/Objectives}

With this analysis we aim to contribute to better characterisation of primary hyperparathyroidism in our setting and support the earlier view regarding greater prevalence of symptomatic disease. Understanding the clinical presentation and its course of PHPT in Indian populations would benefit in future detection and management of PHPT in India.

\section{Materials And Method}

A retrospective study of patients with PHPT who underwent parathyroidectomy at Department of Surgery, S.C.B Medical college and Hospital during January 2004 and December 2011 was carried out. Patients with recurrent primary hyperparathyroidism, secondary and tertiary hyperparathyroidism were excluded.

All related medical data including demographic data, clinical presentations, comorbidities, localizations of abnormal parathyroid glands, operations and their findings, and pathology and complications were reviewed. Demographic data consisted of age, sex, and other diagnosis. Clinical presentations were divided into different physiological systems as skeletal, renal, neuromuscular, neuropsychiatric, gastrointestinal systems, and other Non-specific symptoms. Data on blood chemistry included preoperative and postoperative serum calcium (total and ionized), phosphate, alkaline phosphatase, creatinine, and parathyroid hormone. Imaging modalities for localization and their accuracy when comparing to operative findings were noted.

\section{Iv. Results}

Total of 12 adult cases (m:f =1:5) Age ( 25-50 years)were studied ${ }^{[t a b l e-1]}$. Skeletal manifestations (12),renal calculi(4),and proximal muscle weakness (3) were the most common symptoms of presentation ${ }^{\text {[table-2] }}$ . Biochemical features include hypercalcemia, low inorganic phosphorus, elevated total alkaline phosphatise and high parathyroid hormone. Preoperative localisation was achieved in (12) patients by ultrasound and SESTAMIBI scan ${ }^{[\text {fig.6] }}$ and single adenoma found in $(6)$ cases ${ }^{[\text {fig.7-8]. }}$ parathyroid carcinoma diagnosed in (0) patients. All the cases responded well to surgical excision. Post operative complication of hungry bone disease was seen in(3)and transient hypoparathyroidism developed in ( 2 ) patients. 
Table-1: Age \& Sex Distribution

\begin{tabular}{|l|c|c|}
\hline Age group (years) & Male & Female \\
\hline$<30$ & 0 & 1 \\
\hline $30-40$ & 1 & 3 \\
\hline $40-50$ & 1 & 3 \\
\hline$>50$ & 0 & 3 \\
\hline Total & 2 & 10 \\
& & \\
\hline
\end{tabular}

Table-2: Clinical Manifestations

\begin{tabular}{|l|c|}
\hline \multicolumn{1}{|c|}{ Symptoms } & No of patients \\
\hline Asymptomatic Hypercalcemia & 0 \\
\hline Skeletal system & 12 \\
\hline Renal system & 4 \\
\hline Neuropsychiatric symptoms & 1 \\
\hline Neuromuscular system & 3 \\
\hline Others & 1 \\
\hline Total & 12 \\
\hline
\end{tabular}

\section{Discussion}

PHPT was traditionally described with classical symptoms and signs as painful bones, renal stones, abdominal groans, and psychic moans ${ }^{[6] .}$ However, after measurement of serum calcium levels was included in the routine check-up program in many developed countries, number of asymptomatic cases has been increasing; therefore symptomatic PTHT has become the minority ${ }^{[5]}$.

In many Asian countries, routine testing of serum calcium is still not in a general practice. In Eastern India serum calcium monitoring is also not a routinely practice; therefore our study documented only symptomatic presentation in PHPT.

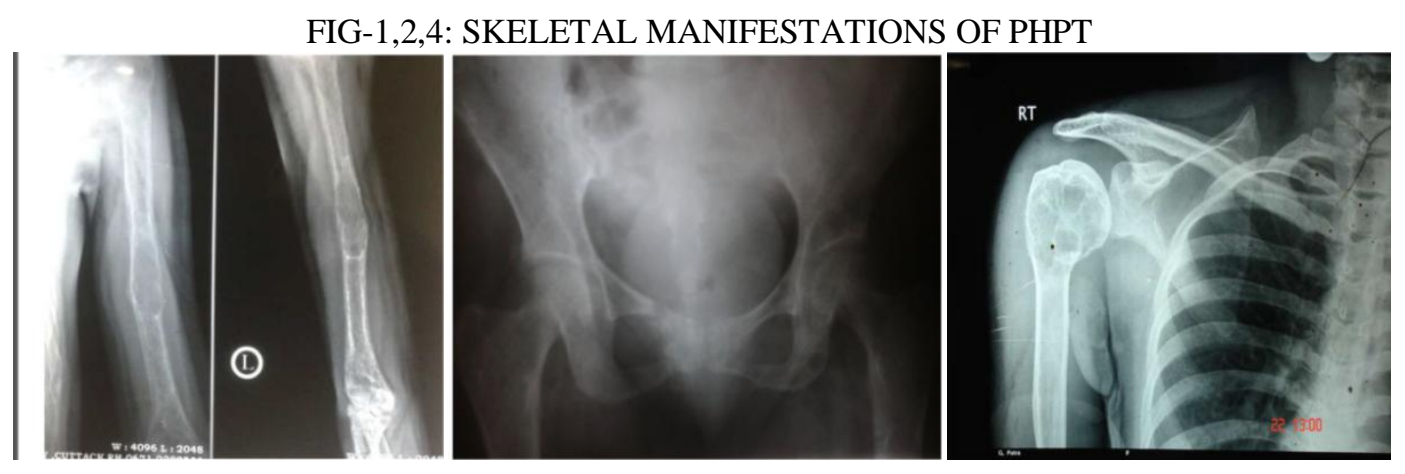

Age distribution of PHPT in our study seems to expand in every age group ( 25 to 50 years) which is similar to Thailand but not Taiwan ${ }^{[7]}$. To design a screening age group of PHPT for early detection in India would need further information. Skeletal and renal related symptoms were the leading clinical presentation of symptomatic PHPT. In this study all the patients presented with pathological fracture ${ }^{[\mathrm{FIG} 1-4], 33.3 \%}$ with renal impairment ${ }^{[\mathrm{fig} .5]}, 25 \%$ with neuromuscular symptoms and $8 \%$ with neuropsychiatric symptoms. These clinical features represented the late diagnosis and showed similar pattern of symptoms to other Asian countries. Although all fractures were re-unioned after parathyroidectomy, the period of treatment was relatively longer than in healthy bone (5-20 months). Anyhow, the renal consequences such as renal calculi and impairment did not improve after parathyroidectomy.

FIG-3: Patient Profile FIG-5: USG Showing Renal Stone

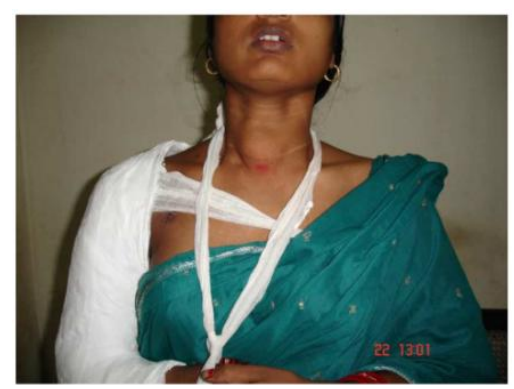

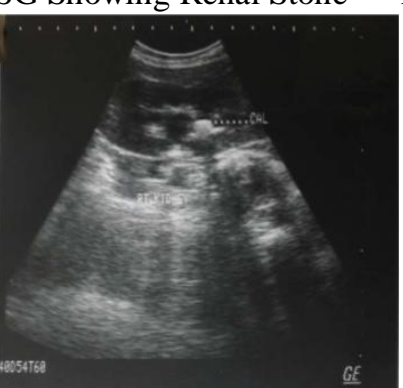

FIG-6: SESTAMIBI Scan of PHPT 
FIG-7, 8: Intra-Operative Findings of PHPT

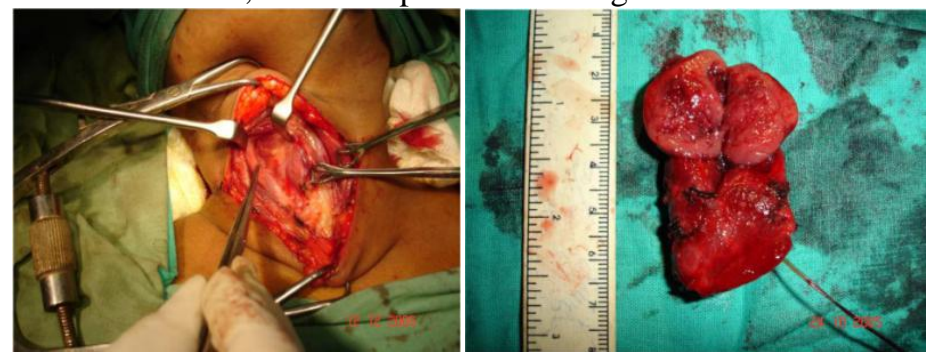

VI. Conclusion

PHPT in our setup is a symptomatic disorder with skeletal and renal manifestation. Lack of universal screening for hypercalcemia and lack of awareness about prevalence and presentation of PHPT among general practitioners led to delayed diagnosis. By improving early detection of PHPT the deadly complications of this curable disease can be avoided.

Patients might benefit from early diagnosis of mild and asymptomatic PHPT only if more efforts are taken to identify these cases. For this to be possible, efforts are needed to incorporate calcium screening in the routine health checkups. The increasing parathyroid awareness among physicians as evidenced by the increase in the number of publications on PHPT will help in diagnosing more cases. Similarly, the introduction of courses (DM and $\mathrm{MCh}$ ) in endocrinology and endocrine surgery by the Indian Medical Council will increase the awareness among practicing clinicians in the area of endocrinology, which in turn will lead to better diagnosis of PHPT. Awareness among the orthopedicians, urologists, nephrologists, and rheumatologists in India also has to increase since in India the majority of patients are initially diagnosed by them. We also see a need for organizing continuing medical education programs targeting these specialists and general practitioners,

especially by those centers who are managing parathyroid diseases .Until then, patients with PHPT in India will continue to be diagnosed late and present with florid manifestations.

\section{References}

[1]. S. Adami, C. Marcocci, and D. Gatti, "Epidemiology of primary hyperparathyroidism in Europe," Journal of Bone and Minera Research, vol.17, no. 2, pp. N18-N23, 2002.

[2]. L. J. Melton III, "Epidemiology of primary hyperparathyroidism,"Journal of Bone and Mineral Research, vol. 6, no. 2, pp. S25-S30, 1991.

[3]. "NIH conference: diagnosis and management of asymptomatic primary hyperparathyroidism: consensus development conference statement," Annals of Internal Medicine, vol. 114, no. 7, pp. 593-597, 1991.

[4]. J. P. Bilezikian, J. T. Potts Jr., G. El-Hajj Fuleihan et al., "Summary statement from a workshop on asymptomatic primary hyperparathyroidism: a perspective for the 21st century," Journal of Clinical Endocrinology \&Metabolism, vol. 87, no. 12, pp. 5353$5361,2002$.

[5]. D. S. Rao, E. R. Phillips, G. W. Divine, and G. B. Talpos, "Randomized controlled clinical trial of surgery versus no surgery in patients with mild asymptomatic primary hyperparathyroidism," Journal of Clinical Endocrinology and Metabolism, vol. 89, no. 11, pp. 5415-5422, 2004.

[6]. E. L. Kaplan, T. Yashiro, and G. Salti, "Primary hyperparathyroidism in the 1990s: choice of surgical procedures for this disease," Annals of Surgery, vol.215, no. 4, pp. 300-317, 1992.

[7]. P.Prasarttong-Osoth, P.Wathanaoran, W.Imruetaicharoenchoke, and S. Rojananin" Primary Hyperparathyroidism: 11-Year Experience in a Single Institute in Thailand" International Journal of Endocrinology Volume 2012, Article ID 952426, 4 pages doi:10.1155/2012/952426 